\title{
Arquitecturas de radio cognitiva: una revisión actual
}

\section{Cognitive radio architectures: a review}

\section{Hans Raúl Márquez Ramos}

Ingeniero Electrónico. Joven Investigador Colciencias 2011,Universidad

Distrital Francisco José de Caldas. Bogotá, Colombia.

Contacto:hans_marquez@hotmail.com

Fecha de recepción: 15 de octubre de 2012

Clasificación del artículo: revisión

Fecha de aceptación: 21 de mayo de 2013

Financiamiento: Colciencias y Universidad Distrital Francisco José de Caldas

Palabras clave: arquitectura de red, radio cognitiva, redes inalámbricas, telecomunicaciones.

Keywords: Network architecture, cognitive radio, wireless network, telecommunications.

\section{RESUMEN}

En años recientes la tecnología en telecomunicaciones ha evolucionado permitiendo el ofrecimiento de mayores servicios a los usuarios, como es el caso de la telefonía móvil. Sin embargo, debido al aumento de estos servicios, el sector de las telecomunicaciones se ha visto enfrentado a un problema creciente que debe solucionarse, referente a la saturación del espectro. Frente a ello se ha planteado como solución más firme la radio cognitiva que enfoca sus esfuerzos en realizar un uso más eficiente del espectro disponible. Este artículo presenta entonces una descripción del estado del arte sobre arquitecturas de radio cognitiva, las soluciones planteadas y las dimensiones espectrales donde se pueden encontrar oportunidades para la transmisión.

\footnotetext{
ABSTRACT

In recent years in telecommunications technology has evolved allowing offering more services to users, such as mobile telephony. However due to the increase of these services, the telecommu-
} 
nications sector has been faced with a growing problem that must be addressed concerning the saturation of the spectrum. Cognitive radio has presented like the best way to solve this problem, focusing their efforts on making more efficient use of the available spectrum. This paper presents then a description of the state of the art on Cognitive radio architectures, the proposed solutions and spectral dimensions where Cognitive Radio can find opportunities for transmission.

\section{INTRODUCCIÓN}

Los crecientes servicios en comunicaciones y la heterogeneidad de las redes han generado la formación de arquitecturas de red que respondan a esta diversidad de tecnologías tales como Personal Digital Assistant (PDA), portátiles y teléfonos inteligentes que están siendo diseñados para hacer uso de los estándares inalámbricos y aprovechar los servicios ofrecidos por esta tecnología, como el caso de la tecnología $3 \mathrm{G}$, que ofrece transmisión de datos y voz, y adicionalmente permite realizar video llamadas.

Este crecimiento en los servicios del sector de las comunicaciones y las nuevas tecnologías dispuestas para el envío y recepción de información, requiere del mejoramiento de la eficiencia en la utilización del espectro inalámbrico, con el fin de que sea una alternativa para evitar la escasez de ancho de banda disponible para la transmisión de la información.

Estudios realizados en Nueva York en septiembre del 2004 sobre mediciones de ocupación del espectro evidencian que las bandas de frecuencias que tienen mayor ocupación son: la banda de 174-216 MHz, con una ocupación aproximada del $77 \%$, seguida por la banda $54-88 \mathrm{MHz}$ con una ocupación del espectro del 52,5\% y la banda 1850-1990 MHz con un 33,8\% de ocupación del espectro [1].

En Dublin (Irlanda), en abril del 2007 se realizaron las mismas mediciones al uso del espectro y en el estudio se observa que donde hay una mayor ocupación del espectro es en la banda 1710-1850 MHz,la cual tiene un porcentaje de ocupación del 38,5\%, seguidade cerca por la banda $1850-1990 \mathrm{MHz}$ con una ocupación del 35,1\%. Estas son las bandas que mayor ocupación del espectro tienen [2].

En otro estudio realizado [3], donde se pone en consideración la banda comprendida entre los $30 \mathrm{MHz}$ y los $3 \mathrm{GHz}$, se evidencia que ciertas bandas de frecuencias no están siendo extensivamente utilizadas, mostrándose por ejemplo que las frecuencias utilizadas para la televisión tan solo usan aproximadamente el $25 \%$ del espectro que tienen licenciado para realizar sus transmisiones, lo cual deja en evidencia que la escasez en la disponibilidad de frecuencias en el espectro se debe primordialmente a la asignación fija de frecuencias.

Con la asignación estática y restrictiva de las bandas de frecuencias en el espectro radioeléctrico, y con la creciente demanda de uso del espectro debido a las nuevas tecnologías emergentes y consecuentes servicios, se está causando un progresivo detrimento en la calidad de servicio (QoS) que se les brinda a los usuarios de tecnologías inalámbricas, como por ejemplo las redes celulares. Esta problemática creciente ha generado investigaciones que buscan posibles soluciones que permitan un uso más eficiente del espectro sin afectar la calidad de servicio, las cuales han arrojado como resultado algunas soluciones como la radio cognitiva, que permite técnicas de acceso dinámicas al espectro [4]. 


\section{DEFINIENDO RADIO COGNITIVA}

En torno a radio cognitiva se han propuesto varias definiciones de acuerdo a la forma como debe funcionar, su capacidad de auto reconfiguración, interacción con su entorno y demás atributos [5][8]. Sin embargo, a continuación se exponen las definiciones más sobresalientes.

En 1999 el Doctor Joseph Mitola III acuñó el término radio cognitiva y lo definió en su artículo Cognitive Radio for Flexible Multimedia Communications como [9], [10]: "A radio that employs model based reasoning to achieve a specified level of competence in radio-related domains". ${ }^{1}$

Porsu parte, la Federal CommunicationsCommission (FCC) realizó la definición de radio cognitiva enfocada al modo de operación de los transmisores de acuerdo con los estímulos externos [11]: "A radio that can change its transmitter parameters based on interaction with the environment in which it operates". ${ }^{2}$

La IEEE USA (Institute of Electrical and Electronics Engineers, Estados Unidos), por su parte, ofreció la siguiente definición [12]:

A radio frequency transmitter/receiver that is designed to intelligently detect whether a particular segment of the radio spectrum is currently in use, and to jump into (and out of, as necessary) the temporarily-unused spectrum very rapidly, without interfering with the transmissions of other authorized users. ${ }^{3}$
El grupo de estudio de radiocomunicaciones de la International TelecommunicationUnion (ITU), en su reporte orientado a Software Defined Radio (SDR), ofrece la siguiente definición sobre radio cognitiva [13]:"A radio or system that senses, and is aware of, its operational environment and can dynamically and autonomously adjust its radio operating parameters accordingly". ${ }^{4}$

\section{CARACTERÍSTICAS DE LA RADIO COGNITIVA}

Las características de la radio cognitiva son las siguientes:

i. Ya sea directa o indirectamente, el dispositivo con radio cognitiva debe ser capaz de adquirir la información del ambiente en el que opera. De acuerdo con esta característica la radio cognitiva puede seleccionar la frecuencia de operación y el formato de trasmisión o también puede actuar como un puente entre 2 sistemas, de manera que reciba la señal en una frecuencia y formato y pueda retransmitir esta señal en la frecuencia y formato adecuados para que sea reconocida por el sistema receptor de la información [14]. La tecnología de radio cognitiva puede facilitar el uso de del espectro a usuarios secundarios y mejorar el acceso a este en zonas rurales.

ii. Modulación adaptativa para modificar las características de transmisión para explotar las oportunidades que se presentan en el espectro.

1 Una radio que emplea el modelo basado en razonamiento para conseguir un nivel de competencia en los dominios relacionados con la radio.

2 Una radio que puede cambiar los parámetros del transmisor basado en la interacción con el ambiente en el cual opera.

3 Un transmisor/receptor de radio frecuencia que es diseñado para detectar inteligentemente ya sea un segmento particular del espectro radial que está siendo utilizado y saltar dentro (y fuera del segmento, de ser necesario) del segmento del espectro que no está siento utilizado temporalmente, sin interferir con la trasmisión de los usuarios autorizados.

4 Una radio o sistema que sense y que este consciente del ambiente en el que opera, y pueda dinámicamente y autónomamente ajustar acordemente sus parámetros de operación. 
La radio cognitiva puede seleccionar la modulación más apropiada para una transmisión en particular, con el fin de permitir la interoperabilidad entre los sistemas. De esta forma, radio cognitiva puede comunicarse con sistemas que hacen uso de esquemas de acceso al canal como TDMA (Acceso al medio por división de Tiempo) y CDMA (Acceso al medio por división de Código) dependiendo del tipo de sistema con el que esté estableciendo la comunicación. Otros usos que se le pueden dar a la modulación adaptativa son la selección del ancho de banda basada en la disponibilidad del espectro y la tasa de transferencia deseada. También se puede pensar en nuevos tipos de modulación en radio cognitiva, como la posibilidad de dividir una señal para que ocupe bandas de frecuencias no contiguas simultáneamente [15].

iii. Capacidad de negociar el espectro con otros usuarios para hacer más eficiente la utilización del espectro.

iv. La radio cognitiva puede estar diseñada en arquitecturas de red centralizada, distribuida, adhoc y redes mesh y estar en capacidad de ser usada tanto para aplicaciones licenciadas como para las que no lo son. De esta forma, las funcionalidades de la radio cognitiva pueden ser aprovechadas por una estación base $B S$ dentro de una red centralizada mesh para coordinar los accesos al medio de cada uno de los usuarios pertenecientes a la red, o puede ser asimismo utilizada en redes $a d h o c$, las cuales no poseen una topología de red definida, donde se pueden conectar 2 dispositivos sin necesidad de un coordinador en la comunicación [16], [17].

v. Capacidad de cambiar rápidamente la frecuencia de operación, ya que se requiere una agilidad en este cambio en los procesos de negociación e intercambio del espectro que se puede dar entre usuarios licenciados y usuarios secundarios, lo cual contribuye de manera directa en la eficiencia del espectro y en la reducción de interferencia co-canal con otros usuarios que hacen uso del espectro en un momento determinado.

vi. Control de potencia en la transmisión o TPC (Trasmit Power Control) es una característicaque permite a un dispositivo, en este caso particular, de radio cognitiva, configurar dinámicamente los niveles de potencia de acuerdo con las exigencias en el proceso de trasmisión. Bajo estas condiciones los dispositivos deben tener en cuenta la cercanía de dispositivos vecinos, la máxima potencia permitida en la localización geográfica o la cantidad mínima de potencia que se requiere en el proceso de trasmisión, para que la información sea enviada de manera confiable, minimizando los posibles errores en la transmisión [18].

vii. La radio cognitiva puede incorporar un mecanismo que permita conocer su ubicación con respecto a la de otros transmisores y a partir de ello seleccionar parámetros adecuados para las transmisiones como la potencia y frecuencia permitida para su localización. Una opción para realizar esta tarea es la geolocalización, que hace uso de GPS para poder determinar la localización geográfica del dispositivo y esta información puede ser introducida a la base de datos que maneje la red, de forma que pueda ser usada por esta para evitar posibles interferencias con otros dispositivos, o asimismo la información puede ser utilizada cuando los métodos de sensado de la red no pueden identificar la localización de usuarios que se encuentren cerca del rango de cobertura de la red [19].

viii. Debe estar en capacidad de tener un mecanismo que permita compartir el espectro con usuarios no licenciados. Al respecto existen mecanismos para compartir el espectro de licencias de servicios no públicos de radio inalámbrica, que 
Tabla 1. Características de radio cognitiva según definiciones dadas de radio cognitiva

\begin{tabular}{|c|c|c|c|c|c|c|c|c|c|c|c|}
\hline Definer & 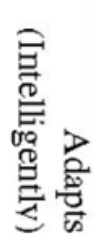 & 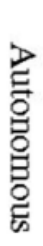 & 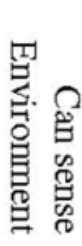 & 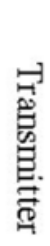 & 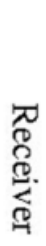 & 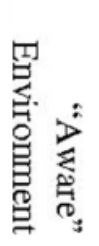 & 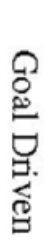 & 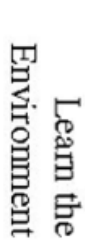 & 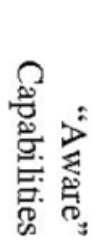 & 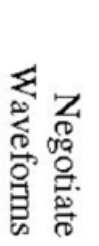 & 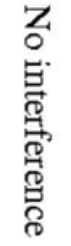 \\
\hline FCC & $\bullet$ & $\bullet$ & $\bullet$ & $\bullet$ & & & & & & & \\
\hline Haykin & $\bullet$ & $\bullet$ & $\bullet$ & • & • & $\bullet$ & $\bullet$ & • & & & \\
\hline IEEE 1900.1 & $\bullet$ & $\bullet$ & $\bullet$ & • & $\bullet$ & & & & & & \\
\hline IEEE USA & $\bullet$ & $\bullet$ & $\bullet$ & $\bullet$ & $\bullet$ & $\bullet$ & & & & & • \\
\hline ITU-R & $\bullet$ & $\bullet$ & • & $\bullet$ & $\bullet$ & $\bullet$ & & & & & \\
\hline Mitola & $\bullet$ & $\bullet$ & $\bullet$ & $\bullet$ & $\bullet$ & $\bullet$ & $\bullet$ & $\bullet$ & $\bullet$ & $\bullet$ & \\
\hline NTIA & $\bullet$ & $\bullet$ & $\bullet$ & $\bullet$ & • & $\bullet$ & $\bullet$ & & & & \\
\hline SDRF CRWG & $\bullet$ & $\bullet$ & • & $\bullet$ & $\bullet$ & & $\bullet$ & & & & \\
\hline SDRF SIG & $\bullet$ & $\bullet$ & • & $\bullet$ & $\bullet$ & $\bullet$ & $\bullet$ & $\bullet$ & $\bullet$ & & \\
\hline VT CRWG & $\bullet$ & $\bullet$ & $\bullet$ & $\bullet$ & $\bullet$ & $\bullet$ & $\bullet$ & $\bullet$ & • & & \\
\hline
\end{tabular}

Fuente: tomada de [14].

realizan esta tarea; sin embargo, se está sujeto a un costo sustancial por el uso del espectro. La radio cognitiva en este sentido posibilitaría el compartir el espectro incurriendo en el menor costo posible por la transacción, automatizando todos o parte de los procesos requeridos para negociar los términos de arrendamiento del espectro. Estos términos se refieren a las frecuencias disponibles, niveles de potencia y tiempo de permanencia en el espectro [3].

En la tabla 1 [14] se detallan las cualidades que debe tener la radio cognitiva de acuerdo a las definiciones dadas por varios autores.

\section{ARQUITECTURAS DE RADIO COGNITIVA}

A continuación se detallan los diferentes tipos de arquitecturas de radio cognitiva que han realizado entes gubernamentales e instituciones de educa- ción superior en respuesta a la eficiencia en el uso del espectro.

\subsection{CR1}

CR1 es la arquitectura desarrollada por Joseph Mitola en su tesis doctoral [20], donde se utiliza el lenguaje de representación de conocimiento de radio RKRL (Radio Knowledge Representation Language) para determinar las adaptaciones de la radio. Este lenguaje representa el conocimiento de radio de un conjunto de lenguajes naturales y modelos basados en computador.

\section{$4.2 \times G$}

El programa de DARPA xG está llevando a cabo una implementación de radios cognitivas, que tiene la iniciativa de autorizar espectro de uso estático, como resultado de una aparente escasez de espectro que puede ser evitado por la correcta 


\section{revisión}

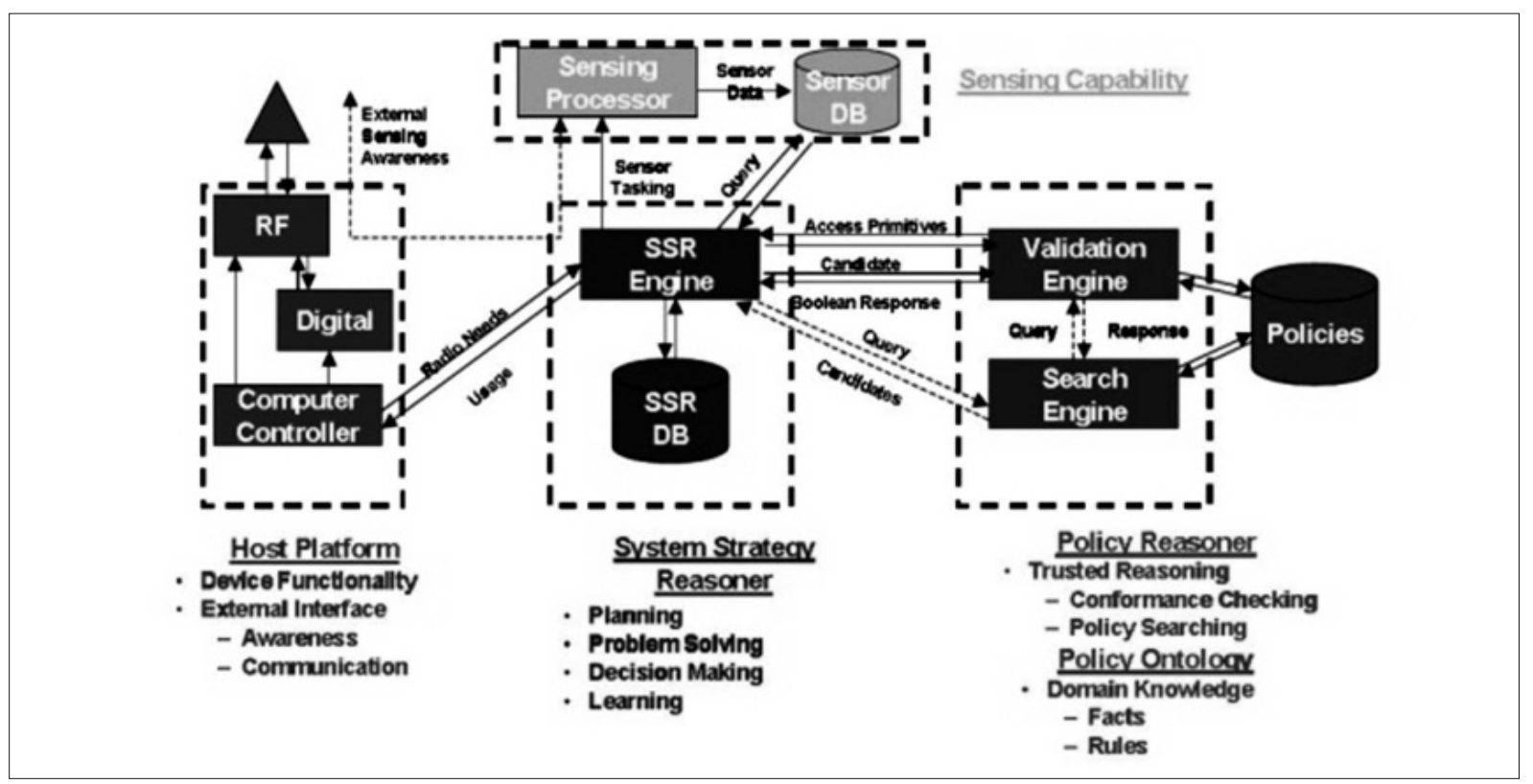

Figura 1. Estructura de $x G$

Fuente: tomada de [21].

aplicación de las técnicas de dinámicas de reparto del espectro. Ha auspiciado igualmente el proyecto "Protocolos de Acceso al Medio para XG (XMAC)", proyecto en el cual se utiliza una arquitectura que relaciona el motor de políticas (policy engine) con el motor de estrategias (System Strategy Reasoner) [21]. En la figura 1 se muestran los componentes de $\mathrm{xG}$.

\subsection{Radio cognitiva biológicamente inspirada}

Tema del trabajo doctoral de Christian Rieser [22], se utiliza en mediciones de canal para construir un modelo oculto de Markov (HMM) de su entorno. Los resultados del HMM a su vez son utilizados por un algoritmo genético para predecir el rendimiento de diferentes combinaciones de componentes de forma de onda para las condiciones de canal observado.

\subsection{CORTEKS}

Radio cognitiva implementada en la Universidad de Virginia Tech. CORTEKS es controlado por un software de políticas definidas que actúa como un usuario secundario del espectro adaptando su frecuencia de operación y modulación para maximizar las posibilidades y tratando en la misma vía de evitar las interferencias que se pudiesen ocasionar con los usuarios primarios. Para determinar la presencia de usuarios primarios utiliza redes neuronales para clasificar las señales [23].

En la figura 2 se muestran los componentes de CORTEKS.

\subsection{Adapt4 XG1}

Adapt4 es un tipo de radio cognitiva y actualmente es un campo de la radio cognitiva XG1. Utiliza un algoritmo propietario denominado Automati Spectrum Adaptation Protocol (ASAP), el cual está diseñado para operar como usuarios secundarios [24]. Tiene características como: selección dinámica de frecuencia, salto de frecuencias y control de potencia en la tras- 


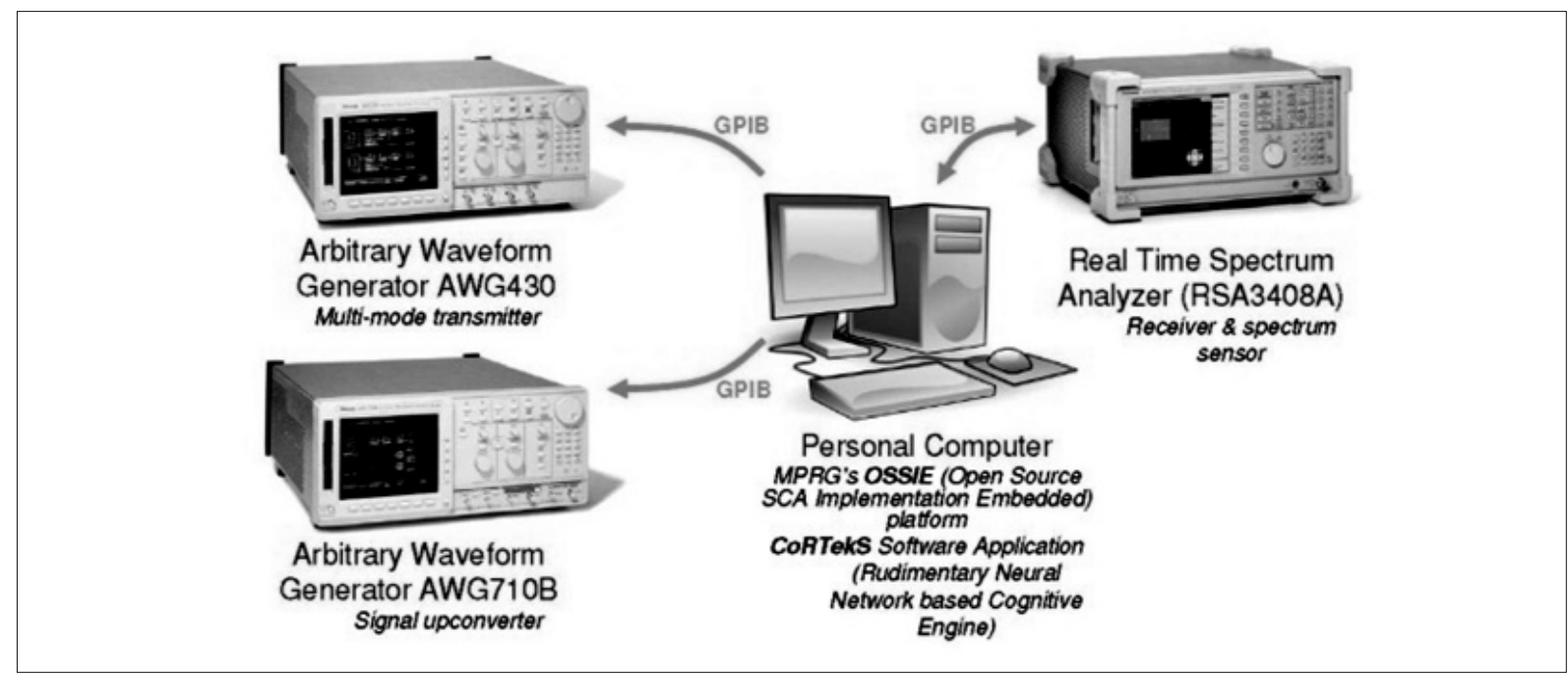

Figura 2. Componentes de CORTEKS

Fuente: tomada de [23].

misión en los casos donde sea posible y minimizar la interferencia para los usuarios primarios.

\subsection{Spectrum pooling}

En [25], [26] se propone la utilización de multiplexación por división de frecuencia ortogonal (OFDM). Esta arquitectura consta de una estación base $\mathrm{xG}$ y usuarios móviles $\mathrm{xG}$. La modulación OFDM es candidata para este trabajo debido a que con este tipo de modulación es posible dejar un grupo de subportadoras sin modular, lo que es conveniente cuando estas subportadoras están siendo utilizadas por los usuarios primarios. La detección de usuarios es realizada mediante el envío periódico, a través de la red, de tramas de detección. Con estas tramas los usuarios móviles actualizan el estado del sensado del espectro. La información de detección es entonces recogida en la estación base.

Los usuarios móvil es modulan un símbolo complejo a máxima potencia en las subportadoras donde los usuarios primarios aparecen. A través de este método la estación base recibe una señal amplificada de todas las subportadoras con los nuevos usuarios primarios.

\subsection{CORVUS}

Esta arquitectura está enfocada en la utilización de usuarios no licenciados o secundarios para sacar ventaja del espectro que no esté siendo utilizado en una banda de frecuencias licenciadas. En [27], [28], CORVUS maneja un grupo de usuarios secundarios denominado SUG, los cuales se comunican entre sí mediante una red ah hoc para coordinar sus comunicaciones.

CORVUS utiliza Spectum Pooling desde el rango de los $\mathrm{MHz}$ hasta los $\mathrm{GHz}$, creando una banda virtual no licenciada. Dentro de ella existen unos canales lógicos dedicados que se utilizan para el intercambio de información de control y sensado denominados UCC (Universal Control Channel) y GCC (Group Control Channel). El primero de ellos se usa para la coordinación entre grupos y el segundo para intercambio interno de cada grupo de información de control y sensado [29]. 
Avances recientes en esta arquitectura plantean un enlace confiable de mantenimiento dentro de CORVUS para mantener la calidad en la comunicación del usuario secundario [30].

En [31] se discute sobre la capa base, así como estrategias de superposición para uso en sistemas de radiocomunicaciones cognitivos, para compartir el espectro. Se presenta, además, el banco de pruebas utilizado para la radio cognitiva, junto con las técnicas basadas en cicloestacionaridad.

\subsection{IEEE 802.22}

Es el primer estándar mundial basado en tecnología de radio cognitiva [32], [33], también conocido como WRAN (Wireless Regional Area Networks). El objetivo prominente es el acceso al ancho de banda en áreas rurales y remotas con un rendimiento comparable a las tecnologías de acceso a banda ancha existentes en la actualidad. Por esta razón que entes gubernamentales, como es el caso de la FCC, estimulan el desarrollo de nuevas tecnologías basadas en radio cognitiva que incrementen la disponibilidad de acceso a banda ancha para estos sectores no atendidos [34], [37].

El estándar hace uso de las bandas de televisión debido a que estas bandas tienen buenas características en cuanto a propagación, permitiendo a potenciales usuarios acceder a los servicios de banda ancha. Por tanto, es un negocio conveniente para los proveedores de servicio de internet inalámbrico WISP (Wireless Internet Service Provider).

Mientras que la mayor parte de desarrollo comercial de radio cognitiva se está realizando en Estados Unidos, el objetivo del estándar IEEE 802.22 es definir un estándar internacional, que pueda operar como un régimen regulatorio. Por tanto, este estándar identifica las bandas de ope- ración desde los 54-862 $\mathrm{MHz}$ para el territorio norteamericano, mientras que aun se encuentra en debate el rango de frecuencia desde los 41$910 \mathrm{MHz}$ para acomodarse a los requerimientos internacionales. De igual forma, el estándar debe acomodarse al ancho de banda de los canales que difieren de 6,7 y $8 \mathrm{MHz}$.

En cuanto a la capacidad del servicio, el estándar determina eficiencias espectrales del orden de los $0,5 \mathrm{bit} /(\mathrm{sec} / \mathrm{Hz})$ hasta los $5 \mathrm{bit} /(\mathrm{sec} / \mathrm{Hz})$, por lo cual para un canal con un ancho de banda de $6 \mathrm{MHz}$ la tasa de transferencia de datos podría ubicarse en el rango de los 3 Mbps hasta los 30 Mbps.

A continuación se muestra un resumen sobre algunas de las especificaciones técnicas que se encuentran en el estándar:

i. Topología de red punto-multipunto.

ii. Máxima potencia irradiada efectiva isotrópica EIRP y radio de la celda: EIRP de 4 Wy la radio de celda de 10 a $100 \mathrm{~km}$ para estaciones base fijas.

iii. Antena Tx / Rx: la estación base BS usa antena omnidireccional. Para el caso del usuario de red la antena $\mathrm{Tx} / \mathrm{Rx}$ es direccional.

iv. Geo-localización: sistemas basados en localización Global Positioning System (GPS).

v. 802.22 soporta la estructura de trama Time Division Duplex (TDD).

vi. Soporta modulaciones QPSK, 16-QAM y 64QAM.

\subsection{DIMSUMnet}

La radio cognitiva DIMSUMnet (Dynamic Intelligent Management of Spectrumfor Ubiquitous Mobile Network) [38], implementa el acceso 
multiplexado estadístico (SMA) al espectro en una banda de acceso coordinado (CAB). La función de SMA es la mejora en la utilización del espectro, mientras que $\mathrm{CAB}$ mejora la eficacia del acceso a espectro y la equidad. CAB utiliza una parte contigua del espectro reservado por las autoridades reguladoras. Un broker o corredor de espectro permanentemente maneja el CAB y lo arrienda de acuerdo con las solicitudes. DIMSUMnet utiliza un sistema centralizado, un mecanismo de intermediación de red regional que tiene como objetivo mejorar significativamente la utilización del espectro y reducir la complejidad y los requerimientos de agilidad del sistema implementado. La estación base se registra con el administrador designado de su red de acceso (RANMAN), el cual a su vez negocia un contrato de arrendamiento de una porción apropiada del espectro con el broker de manejo e información del espectro (SPIM). Si el arrendamiento es exitoso, el RANMAN configura el espectro arrendado en la estación base. La estación base envía la información recibida del RANMAN a sus usuarios para la configuración del cliente [39]. Trabajos recientes se enfocan en el precio del espectro y las funciones de localización del espectro para los brokers del espectro [40].

En [41], los autores investigan las cadenas de Markov en tiempo continuo para acceso dinámico al espectro en redes inalámbricas de espectro abierto, es decir, bandas de frecuencias no licenciadas, y se consideran tanto los modelos de colas como los de no colas. Hacen uso de un modelo antropológico para el acceso al medio, el cual usa solamente la información local, denominado homo egualis (HE). Los resultados analíticos se derivan de los modelos de Markov.

\subsection{Kuar}

En [42], los autores presentan una plataforma de desarrollo de radio definida por software

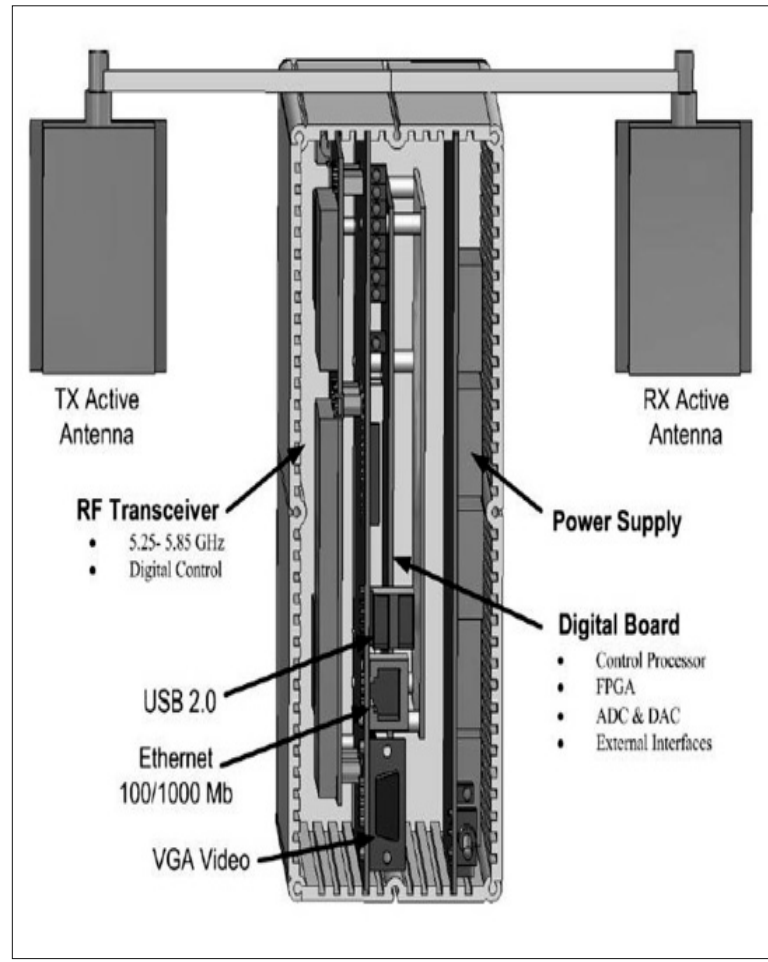

Figura 3. Componentes de KUAR

Fuente: tomada de [43].

portátil, potente y flexible denominada KUAR (Kansas University Agile Radio). El propósito de esta plataforma es permitir la investigación avanzada en las áreas de redes de radio inalámbricas, acceso dinámico al espectro, y radios cognitivas. Describe en detalle los requerimientos en hardware y herramientas de software, y presenta un ejemplo de aplicación de la modulación de KUAR, medición del espectro, estimación de canal, y la rápida configuración y adaptación de KUAR. En la figura 3 se muestra la estructura de KUAR.

\subsection{KNOWS}

Este sistema detecta los huecos en el espectro en la banda de frecuencias de televisión haciendo uso de sensado cooperativo. Emplea un esquema distribuido denominado b-SMART, el cual 


\section{revisión}

dinámicamente ajusta la frecuencia de operación, el tiempo de ocupación en el canal asignado y el ancho de banda de la comunicación, basado en el conocimiento de huecos espectrales disponibles en el espectro. A partir de este conocimiento, KNOWS provee a cada usuario un segmento del ancho de banda disponible.

KNOWS hace uso de un nuevo esquema de control de acceso al medio denominado CMAC, basado en el protocolo MAC. A diferencia de este último, CMAC incorpora una mejora en los mecanismos RTS y CTS mostrados en el estandar IEEE 802.11 y en vez de reservar tiempo de trasmisión en el aire en un canal, reserva segmentos vacíos de frecuencias en el espectro [44].

En la figura 4 se muestran los componentes de KNOWS:

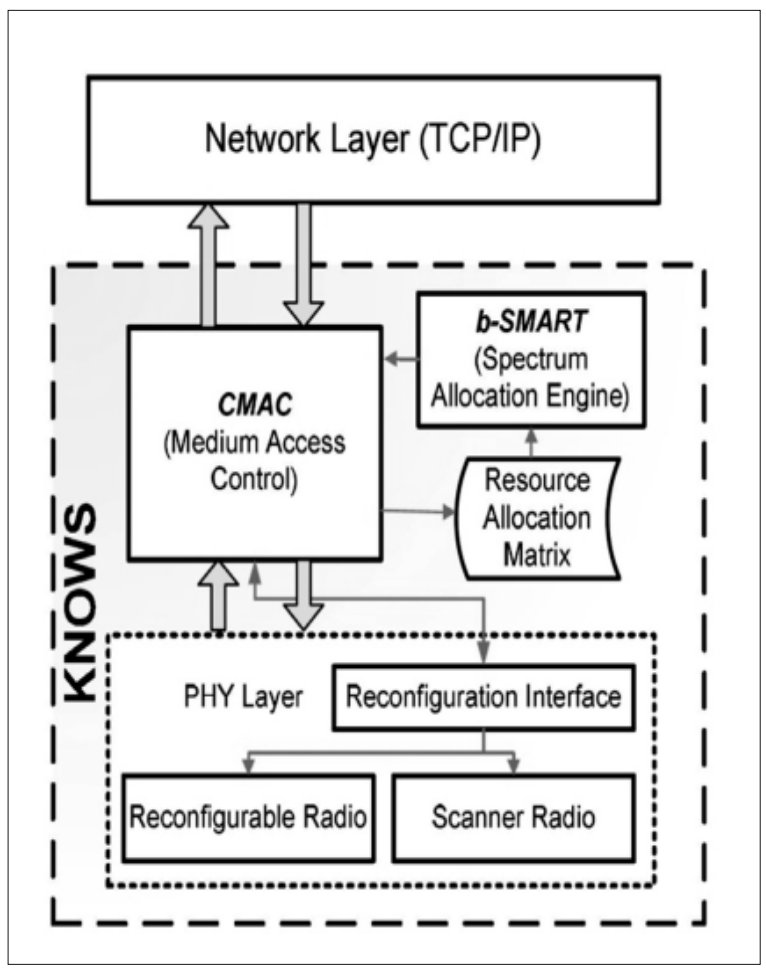

Figura 4. Componentes de KNOWS

Fuente: tomada de [44].

\subsection{DRiVEProject}

El proyecto europeo de radio dinámica para servicios IP en ambientes vehiculares o DRiVE (Dynamic Radio for IP Services in Vehicular Environments), enfoca sus esfuerzos enproveer espectro dinámico en redes donde convergen varias tecnologías heterogéneas, haciendo uso de un canal común de coordinación [45].

El proyecto Over DRiVE fue el siguiente paso del proyecto DRiVE. Este proyecto apunta al desarrollo y coordinación de UMTS (Universal Mobile Telephone Standard) en las redes de radio cognitiva de una red híbrida, para asegurar la eficiencia del espectro para los servicios de multimedia en dispositivos móviles [46].

En la figura 5 se muestra la arquitectura de red de DRiVE:

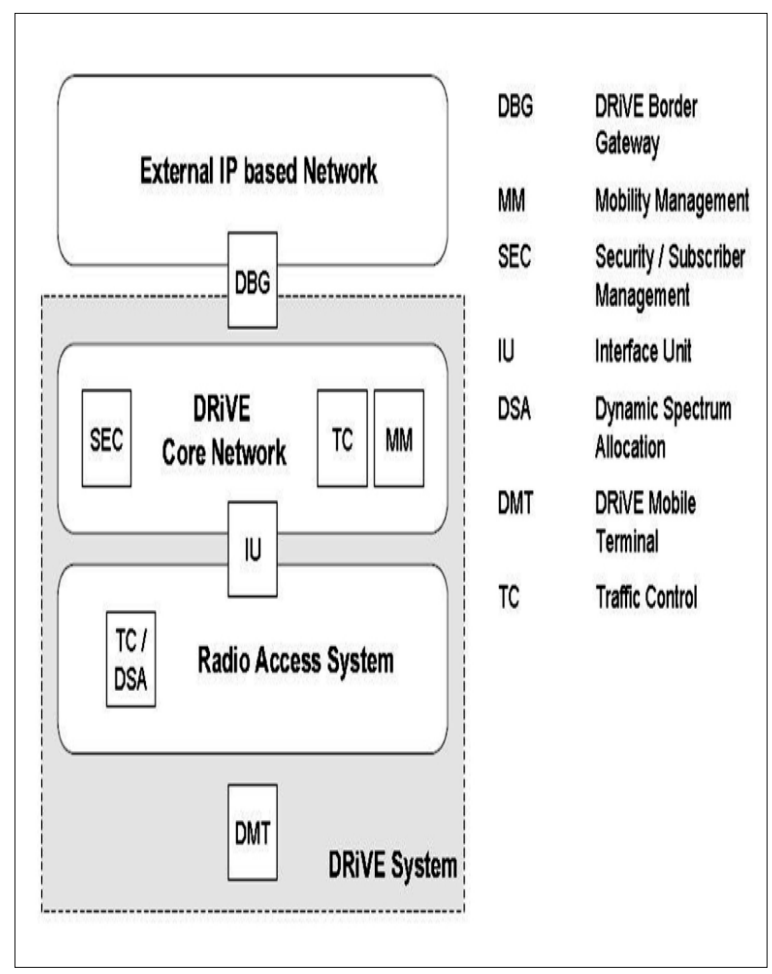

Figura 5. Arquitectura de red de DRiVE

Fuente: tomada de [45]. 


\subsection{Flex}

En [47] los autores proponen FLEX, una arquitectura distribuida para Access point de WiFi, la cual realiza la localización del espectro y eficientemente se adapta a las demandas de tráfico dinámico, de forma que se maximice la satisfacción del usuario.

\subsection{OCRA network}

En [48], la red OCRA considera todos los escenarios de implementación posibles sobre las redes heterogéneas $\mathrm{xG}$ y desarrolla operaciones en la capa cruzada para el acceso al espectro dinámico basado en OFDM. Para la decisión de espectro y el handoff del espectro, la red OCRA provee un nuevo concepto basado en OFDM, el cual consiste en el manejo del espectro en ambientes heterogéneos. A partir de la estructura de la capa física (PHY), se propone una estructura de modo dual de espectro compartido, la cual habilita el acceso a las redes existentes, así como la coordinación entre los usuarios XG.

En [49] se proponen protocolos MAC cognitivos descentralizados que permiten a los usuarios secundarios ser autónomos al momento de buscar oportunidades espectrales, sin tener que estar controlado por un coordinador del espectro o un canal de comunicación dedicado. Se desarrolla asimismo un marco analítico para el acceso al espectro oportunista basado en la teoría del proceso de decisión de Markov Parcialmente Observable (POMDP). Este enfoque de decisión teórica integra el diseño de protocolos de acceso al espectro en la capa MAC con la detección del espectro en la capa física y estadísticas del tráfico determinadas por la capa de aplicación de la red primaria. Sin mensajes de intercambio de control entre el transmisor secundario y el receptor debido a los protocolos descentralizados, asegura saltos síncronos en el espectro entre ellos en presencia de ambientes con colisión y errores en el sensado del espectro.

En [50] los autores diseñan una radio cognitiva que puede coexistir con múltiples canales paralelos WLAN respetando al mismo tiempo una limitación de interferencia. La interacción entre los 2 sistemas, la cual se caracteriza por la medición y la coexistencia, es mejorada por la predicción del comportamiento de WLAN basadas en un modelo de cadena de Markov de tiempo continuo. El acceso al medio cognitivo (CMA) se deriva de este modelo por la recomposición del problema como uno de los procesos de decisión de Markov limitados. La radio cognitiva considerada en este artículo es basada en saltos de frecuencia con una capa física similar a la utilizada por Bluetooth. Esto permite realizar comparaciones conceptuales de coexistencia entre Bluetooth y WLAN.

\subsection{SPARTA}

En [51] los autores proponen la SPARTA, una nueva arquitectura DSA que provee un eficiente y estable uso del espectro integrando la planificación proactiva con la adaptabilidad reactiva. SPARTA introduce un novedoso algoritmo de control de admisión estadístico que proactivamente evita la congestión en la demanda por el espectro, mientras lidia con la interferencia. Esta arquitectura permite determinar el volumen de la demanda soportada por cada Access Point basada en sus estadísticas y la condición de interferencia local.

En [52] se describe un modelo concreto para una radio cognitiva genérica para utilizar un motor de aprendizaje. El objetivo es incorporar los resultados del aprendizaje motor en un motor basado en razonamiento de cálculo, de manera que las radios pueden recordar las lecciones aprendidas en el pasado y actuar con rapidez en el fu- 
turo. También investigan las diferencias entre el razonamiento y el aprendizaje, y los fundamentos de cuando una aplicación particular requiere aprender, y cuando un razonamiento simple es suficiente. La arquitectura básica es consistente con los motores cognitivos observados en la investigación en IA. El objetivo de este artículo no es proponer nuevos algoritmos de aprendizaje de máquina, sino más bien formalizar su solicitud a la radio cognitiva y desarrollar un marco desde el cual pueden ser útiles.

En [53] se presenta una nueva metodología emergente para la inferencia estadística que mejora algunas deficiencias del algoritmo EM. Esta metodología se denomina aproximación variacional y se puede utilizar para resolver los complejos modelos bayesianos donde el algoritmo EM no se puede aplicar. La inferencia bayesiana basada en la aproximación variacional ha sido ampliamente utilizada por la comunidad de aprendizaje de la máquina desde los años noventa, cuando se introdujo por primera vez.

En [54] se propone la evaluación del rendimiento de las redes de radio cognitiva tomando en cuenta factores como las métricas utilizadas por las redes, algoritmos utilizados en el proceso cognitivo y de acuerdo con esto los autores proponen los resultados de acuerdo a los escenarios planteados.

\section{RETOS EN INVESTIGACIÓN DE RADIO COGNITIVA}

Los desafíos de radio cognitiva pueden ser varios, debido a que se abarcan temas desde el sensado del espectro hasta decisiones de movilidad en este, teniendo en cuenta esquemas de acceso al medio y tipo de redes en las cuales interactúa la radio cognitiva. Por tanto, en este apartado se describirán brevemente estos desafíos.

\subsection{Sensado del espectro}

El proceso de sensado no puede realizarse al tiempo en que se envía la información entre usuarios de radio cognitiva, por consiguiente, si se requiere desarrollar acciones de sensado, los usuarios deben detener las transmisiones para realizar sensado, afectando la eficiencia del espectro. Con base en esto sería deseable desarrollar algoritmos de sensado quere duzcan el tiempo de sensado del espectro mientras mejoran la precisión en el proceso de sensado.

\subsection{Espectro compartido}

En trabajos realizados en radio cognitiva se hacen suposiciones que los usuarios secundarios conocen de antemano como la localización y el nivel de potencia de la transmisión de los usuarios primarios, lo cual permite realizar los cálculos de interferencia de manera fácil. Sin embargo, esta suposición no siempre es cierta para algunas redes de radio cognitiva.

\subsection{Procesos de aprendizaje}

Debido a las complejidades inherentes de la radio cognitiva, sería deseable habilitar en los dispositivos que hagan uso de radio cognitiva un proceso de aprendizaje que tome en cuenta las decisiones tomadas en el pasado para mejorar su comportamiento dentro de la red en decisiones futuras.El diseño de este tipo de algoritmos representa un gran desafío, debido a que se debe encontrar qué mediciones deben ser necesarias para desarrollar este proceso de aprendizaje.

\subsection{Esquemas de control de Acceso al Medio}

Aunque el grupo de investigación del estándar IEEE 802.22 está trabajando en el desarrollo de un protocolo de control de Acceso al Medio (MAC), 
otras investigaciones han desarrollado esquemas que no se adecuan al estándar. Por ejemplo, los esquemas de control de acceso al medio distribuidos para redes CRAHN no están del todo cubiertos. En el estándar IEEE 802.11 se tratan algunos temas del manejo inteligente del espectro, como se muestra en IEEE $802.11 \mathrm{k}$, pero este se limita a la operación en la banda ISM.

\section{CONCLUSIONES}

Los desarrollos de arquitecturas para radio cognitiva han sido varios, en la búsqueda de diseñar las mejores alternativas para encontrar oportunidades del espectro mientras minimizan la inter- ferencia en este proceso. Dichas arquitecturas abarcan desde el proceso de sensado hasta los esquemas de acceso al medio para desarrollar sus transmisiones; sin embargo, algunas están diseñadas de manera centralizada, es decir, basándose en un coordinador del espectro que hace la asignación respectiva de las frecuencias disponibles, mientras que otras arquitecturas optan por la forma descentralizada.

Pese a que se han realizado grandes aportes en radio cognitiva aún se presentan grandes desafíos que deben ser tenidos en cuenta en las arquitecturas para hacerlas más óptimas en el proceso de sensado, análisis y decisión sobre el espectro.

\section{REFERENCIAS}

[1] M.A. McHenry, "NSF Spectrum Occupancy Measurements Project Summary", Shared spectrum co. report, Vienna, USA Aug. 2005.

[2] T. Erpek, "Dublin Ireland Spectrum Occupancy Measurements Project Summary", Shared spectrum co. report, Vienna, USA, Nov. 2007.

[3] FCC, ET Docket No. 03-222 Notice of proposed rule making and order, Washington December 2003.

[4] I. Akyildiz, W.Y.Lee, M.C.Vuran, and S. Mohanty, "A survey on spectrum management", in Cognitive radio networks. IEEE Communications Magazine, 2008, pp. 40 -48.

[5] K. Nolan and J. Grosspietsch, "Cognitive Radio WG", SDR Forum, Brussels, Belgium, Powerpoint Presentation, September 14th 2005.

[6] S. Haykin, "Cognitive Radio: BrainEmpowered Wireless Communications",
IEEE Journal on Selected Areas in Communications, vol. 23, no. 2, Feb. 2005.

[7] IEEE Standard P1901.1/D01 (v 0.16), Standard Definitions and Concepts for Spectrum Management and Advanced Radio System Technologies (DRAFT), June 2, 2006.doi: 10.1109/IEEESTD.2008.4633734

[8] D. Maldonado, L. Bin, A. Hugine, T.W. Rondeau, and C.W. Bostian, "Cognitive radio applications to dynamic spectrum allocation: a discussion and an illustrative example", 2005.

[9] J. Mitola, III, "Cognitive Radio for Flexible Multimedia Communications", Mobile Multimedia Communications, 1999 IEEE International Workshop on, pp. 3-10, 1999.

[10] J. Mitola, Cognitive radio - model-based competence for software radios, Licentiate Thesis, KTH, Stockholm (September 1999).

[11] FCC. ET Docket No. 03-108. Facilitating Opportunities for Flexible, Efficientand Re- 
liable Spectrum Use Employing Cognitive

Radio Technologies, Washington,Marzo 11, 2005.

[12] IEEE USA Position"Mejoramiento en el uso del espectro a través de tecnología de radio cognitiva", [En línea]. Disponible [noviembre 13, 2003]: http://www.ieeeusa. org/policy/positions/cognitiveradio.asp

[13] J. D. Poston, W. D. Horne, M. G. Taylor, and F. Z. Zhu, "Ontology-based reasoning for context- aware radios: insights and findings from prototype development",In New Frontiers in Dynamic Spectrum Access Networks, 2005. DySPAN 2005. 2005 First IEEE International Symposium on (pp. 634-637). IEEE..

[14] J.O. Neel, "Analysis and design of cognitive radio networks and distributed radio resource management algorithms", Ph.D. dissertation, Virginia Polytechnic Institute and State University, Sept. 2006.

[15] S. Seidel, R. Breinig y R. Berezdivin, "Adaptive Air Interface Waveform for Flexibility and Performance in Commercial Wireless Communications Systems",In World Wireless Research Forum, Mar. 2002.

[16] P. Santi, "Topology control in wireless ad hoc and sensor networks", ACM Computing Surveys (CSUR), vol. 37, no 2, pp. 164194, 2005.

[17] A. Sgora, D. Vergados, y P. Chatzimisios, "IEEE 802.11 s Wireless Mesh Networks: Challenges and Perspectives", Mobile Lightweight Wireless Systems, pp. 263-271, 2009.

[18] M. Gast, 802.11 wireless networks: the definitive guide, 2a. ed., O'Reilly Media, Incorporated, Grecia 2005.
[19] N.C. Karmakar, N. C. Handbook of smart antennas for RFID systems, Nueva York, Wiley, 2010.

[20] J. Mitola III, "Cognitive Radio: An Integrated Agent Architecture for Software Defined Radio", Ph.D. Dissertation Royal Institute of Technology, Stockholm, Sweden, Mayo 2000.

[21] R. Ramanathan y C. Partridge, "Next generation $(\mathrm{xG})$ architecture and protocol development (XAP)".DARPA, New York, Tech Rep. AFRL-IF-RS-TR-2005-281, 2005.

[22] C. Rieser, "Biologically Inspired Cognitive Radio Engine Model Utilizing Distributed Genetic Algorithms for Secure and Robust Wireless Communications and Networking”, Ph.D. Dissertation, Virginia Tech, Aug. 2004.

[23] J.O. Neel, "Analysis and design of cognitive radio networks and distributed radio resource management algorithms", Ph.D. Dissertation, Virginia Polytechnic Institute and State University, Sept. 2006.

[24] Ground-breaking Technology Adapt4 technology. [En línea]. Disponible:http://www. adapt4.com/adapt4-technology.php.

[25] T.A. Weiss, J. Hillenbrand, A. Krohn, F.K Jondral, Efficient signaling of spectral resources in spectrum pooling systems, inProc. 10th Symposium on Communications and Vehicular Technology (SCVT), Nov. 2003.

[26] T.A. Weiss, and F.K. Jondral, Spectrum pooling: an innovative strategy for the enhancement of spectrum efficiency, IEEE Radio Communication Magazine, vol.42, no. 3, pp. 8-14, Mar. 2004. 
[27] R.W. Brodersen, A. Wolisz, D. Cabric, S.M. Mishra, D. Willkomm, Corvus: a cognitive radio approach for usage of virtual unlicensed spectrum, California, Berkeley Wireless Research Center (BWRC) White paper, 2004.

[28] D. Cabric, S.M. Mishra, D. Willkomm, R. Brodersen, A. Wolisz, A Cognitive radio approach for usage of virtual unlicensed spectrum, in Proc. 14th IST Mobile and Wireless Communications Summit, Jun. 2005.

[29] S.M. Nishra, D. Cabric, C. Chang, D. Willkomm, B. Schewick, A. Wolisz, and R.W. Brodersen, A real time cognitive radio testbed for physical and link layer experiments, in Proc. IEEE DySPAN 2005, Nov. 2005, pp. 562-567.

[30] D. Willkomm, J. Gross, and A. Wolisz, Reliable link maintenance in cognitive radio systems, in Proc. IEEE DySPAN 2005, Nov. 2005, pp. 371-378.

[31] D. Cabric, I. D. ODonnell, M. S.-W. Chen, and R. W. Brodersen, "Spectrum Sharing Radios", IEEE Circuits and Systems Magazine, vol. 6, no. 2, pp. 30-45, 2006.

[32] C. Cordeiro, K. Challapali, D. Birru, and S. Shankar, IEEE 802.22: the first worldwide wireless standard based on cognitive radios, in Proc. IEEE DySPAN2005, Nov.2005, pp. 328-337.

[33] IEEE 802.22 Working group on wireless regional area networks, Enabling Spectrum Sharing and Rural Broadband Wireless Access Using Cognitive Radio Technology in White SpacesRecipient of the IEEE SA Emerging Technology Award. IEEE 802.22 Working group on wireless regional area networks, [En línea]. Disponible: http:// www.ieee802.org/22/
[34] Federal Communications Commission (FCC), "Notice of Proposed Rule Making," ET Docket no. 04-113, May 25, 2004.

[35] Federal Communications Commission (FCC), "Report and Order AND Memorandum Opinion and Order", ET Docket no. 05-56, March 16, 2005.

[36] Federal Communications Commission (FCC), "Report and Order", ET Docket no. 05-57, March 11, 2005.

[37] K. Challapali, D. Birru, and S. Mangold, "Spectrum Agile Radio for Broadband Applications", EE Times In-Focus, August 23, 2004. [En línea]. Disponible:http://eetimes.com/design/other/4003528/SpectrumAgile-Radio-for-Broadband-Applications

[38] M.M. Buddhikot, P. Kolody, S. Miller, K. Ryan, J. Evans, DIMSUMNet: new directions in wireless networking using coordinated dynamic spectrum access, In Proc. IEEE WoWMoM2005, June2005, pp. 78-85.

[39] T. Kamakaris, M.M. Buddhikot, R. Iyer, A case for coordinated dynamic spectrum access in cellular networks, inProc. IEEE DySPAN 2005, Nov.2005, pp. 289- 298.

[40] M.M. Buddhikot, and K. Ryan, Spectrum management in coordinated dynamic spectrum access based cellular networks, inProc. IEEE DySPAN 2005, Nov. 2005, pp. 299-307.

[41] Y. Xing and R. Chandramouli, "Dynamic Spectrum Access in Open Spectrum Wireless Networks", IEEE Journal on Selected Areas in Communications, vol. 24, no. 3, pp. 626-637, Mar. 2006.

[42] G. J. Mindenetál, "An Agile Radio for Wireless Innovation", IEEE Communica- 
tions Magazine, vol. 45, no. 5, pp. 113-121, May 2007.

[43] G.J. Minden et ál, "KUAR A Flexible Software-Defined Radio Development Platform" in New Frontiers in Dynamic Spectrum Access Networks, 2007. DySPAN 2007, 2nd IEEE International Symposium, pp. 428-439.

[44] Y. Yuan, P.Bahl,R. Chandra,P.A. Chou,J.I. Ferrell,T.Moscibroda,S.Narlanka, and Y.Wu,"KNOWS: Cognitive Radio Networks Over White Spaces", New Frontiers in Dynamic Spectrum Access Networks, 2007. DySPAN 2007. 2nd IEEE International Symposium, pp.416-427, 17-20 April 2007.

[45] L. Xu, R. Tonjes, T. Paila, W. Hansmann, M. Frank, and M. Albrecht, "DRiVEing to the internet: dynamic radio for ip services in vehicular environments", in Proc. of 25th Annual IEEE Conference on Local Computer Networks, Nov. 2000, pp. 281-289

[46] D. Grandblaise, D. Bourse, K. Moessner, and P. Leaves, Dynamic spectrum allocation (DSA) and reconfigurability, in Proc. Software-Defined Radio (SDR) Forum, Nov. 2002.

[47] L. Yang, L. Cao, H. Zheng, and E. Belding, "Trafc-Aware Dynamic Spectrum Access", in 4th International Wireless Internet Conference, WICON, Nov. 2008.

[48] I.F. Akyildiz, and Y. Li, OCRA: OFDMbased cognitive radio networks, Broadband and Wireless Networking Laboratory Technical Report, March 2006.

[49] Q. Zhao, L. Tong, A. Swami, and Y. Chen, "Decentralized Cognitive MAC for Op- portunistic Spectrum Access in Ad Hoc Networks: A POMDP Framework", IEEE Journal on Selected Areas in Communications, vol. 25, no. 3, pp. 589-600, Apr. 2007.

[50] S.Geirhofer, L. Tong, and Sadler, B.M., "Cognitive Medium Access: Constraining Interference Based on Experimental Models", Selected Areas in Communications, IEEE Journal, vol. 26, no.1, pp. 95-105, Jan. 2008

[51] L. Cao, H. Zheng, "Stable and Efficient Spectrum Access in Next Generation Dynamic Spectrum Networks", INFOCOM 2008. The 27th Conference on Computer Communications. IEEE, IEEE 802.22 Working group on wireless regional area networks, Enabling Spectrum Sharing and Rural Broadband Wireless Access Using Cognitive Radio Technology in White Spaces Recipient of the IEEE SA Emerging Technology Award, pp.870-878, 13-18 Abril 2008.

[52] C. Clancy, J. Hecker, E. Stuntebeck, and T. O'Shea, "Applications of Machine Learning to Cognitive Radio Networks", IEEE Wireless Communications, vol. 14, no. 4, pp. 47-52, Aug. 2007.

[53] D. G. Tzikas, A. C. Likas, and N. P. Galatsanos, "The Variational Approximation for Bayesian Inference",IEEE Signal Processing Magazine, vol. 25, no. 6, pp. 131-146, Nov. 2008.

[54] Y. Zhao, S. Mao, J. O. Neel, and J. H. Reed, "Performance Evaluation of Cognitive Radios: Metrics, Utility Functions, and Methodology", Proceedings of the IEEE, vol. 97, no. 4, pp. 642-659, Apr. 2009. 